\title{
A novel helper-dependent adenovirus-based cell culture model for Hepatitis $C$ virus replication and production
}

Xiaojun Zhou ${ }^{1,2}$, Yang Zeng ${ }^{1 \dagger}$, Junfeng $\mathrm{Li}^{1}$, Yan Guo ${ }^{1}$, Yuanhui $\mathrm{Fu}^{3}$, Jinsheng $\mathrm{He}^{3}$, Shihui Sun ${ }^{1 *}$ and Yusen Zhou ${ }^{1 *}$

\begin{abstract}
Background: By using the hepatitis C virus (HCV) genotype 2a JFH-1 or its chimeric strains, a HCV infection system has been previously developed through several methods- such as in vitro-transcribed JFH1-RNA transfection or stable transfection of the JFH1 cDNA into human hepatoma Huh-7 cell line or its derivatives. However, other reliable methods for delivery of the HCV genome into cells are still worth trying. The helper-dependent adenovirus (HDAd) is devoid of all viral coding sequences and has a package capacity of $37 \mathrm{~kb}$, which is suitably large for the delivery of the HCV genome. Here we report a new method for delivery of the HCV genome into Huh-7 and HepG2 cells by using the HDAd vector.

Results: Our results demonstrated that the infection of Huh-7 cells with the HDAdJFH1 virus led to efficient HCV replication and virion production. We found that the HCV viral RNA levels could reach 107 copies per milliliter (ml) in the culture medium. HDAdJFH1-infected Huh-7 cells could be cultured for 8 passages with the culture medium remaining infectious for naive Huh-7 cells throughout this period. This infection system proved effective for evaluating the anti-HCV effects of IFN-a in Huh-7 cells. Co-infection of HepG2 cells with the HDAdJFH1 and HDAdmiR-122 virus also resulted in HCV expression and replication.
\end{abstract}

Conclusion: This is the first report of an HDAd-based strategy for HCV replication and production in vitro.

Keywords: Hepatitis C virus, Cell-culture model, Helper-dependent adenovirus

\section{Background}

Hepatitis C virus (HCV) is a $9.6 \mathrm{~kb}$ positive-strand RNA virus and a member of the flavivirus family [1]. It is estimated that approximately 170 million people worldwide are persistently infected by $\mathrm{HCV}$, which can result in hepatic fibrosis, cirrhosis and hepatocellular carcinoma [2]. Current interferon- $\alpha$ based therapy, in combination with ribavirin, has limited efficacy in only approximately $50 \%$ of patients and has severe side effects [3]. HCV replication takes place in the cytoplasm, and the $9.6 \mathrm{~kb}$ RNA genome encodes a polyprotein localized to the rough endoplasmic reticulum (ER), where it is cleaved into at least 10 structural (C, E1, E2, and P7) and

\footnotetext{
* Correspondence: sunsh01@163.com; yszhou@nic.bmi.ac.cn

${ }^{\dagger}$ Equal contributors

'State Key Laboratory of Pathogen and Biosecurity, Beijing Institute of Microbiology and Epidemiology, Beijing, China

Full list of author information is available at the end of the article
}

nonstructural (NS2, NS3, NS4A, NS4B, NS5A, and NS5B) proteins that play important roles in virus replication, assembly and pathogenesis [4].

$\mathrm{HCV}$ research has been hampered by the lack of adequate in vitro and in vivo model systems. Replicons have been utilized for studying HCV RNA replication, but these are not useful for studying aspects of virion production and infection [5]. In 2005, researchers discovered a genotype $2 \mathrm{a}$ isolate JFH1 from a Japanese patient with fulminant hepatitis that could exhibit complete the virus life cycle after transfection of in vitro transcribed full-length JFH1 RNA into Huh-7 or Huh7.5 cells. This system is also able to produce infectious viral particles in cell culture (HCVcc) [6,7]. Furthermore, it was found that stable human hepatoma cell lines containing a chromosomally integrated cDNA copy of

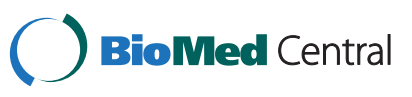


the JFH1 genome with a hepatitis delta virus ribozyme at the $3^{\prime}$ end can constitutively produce infectious viral particles [8]. These methods have proven to be effective in generating infectious $\mathrm{HCV}$ cell culture models in Huh-7 cell line and its derivatives.

In contrast to Huh-7 cells, the hepatocellular carcinoma derived HepG2 cells polarize and would thus permit the investigation of how cell polarization impacts the HCV life cycle [9]. However, HepG2 cells does not express endogenous miR-122, a liver-expressed miRNA which is required to support HCV RNA replication [10], and weakly supports HCV replication [11]. Although a recent study has indicated that HepG2 cells expressing miR-122 can support the entire HCV life cycle [11], the efficiency of $\mathrm{HCV}$ replication and virion production still needs increasing. Thus other potential methods besides transfection for delivery of the $\mathrm{HCV}$ genome into cells are still worth trying especially when the cells, such as HepG2 cells, possess a relatively lower transfection efficiency.

Adenoviruses (Ads) are non-enveloped double-stranded DNA viruses, which can mediate efficient transduction and expression of foreign genes in cells [12]. The helperdependent adenovirus (HDAd) possesses the same ability to deliver foreign DNA into cells as earlier generation adenoviruses (Ads); furthermore, HDAd vectors are devoid of all viral coding sequences and have cloning capacities of up to $37 \mathrm{~kb}$ [13], which makes it possible to introduce large genes into cells using HDAd vectors. Lacking all viral coding sequences, it displays only minimal immunogenicity and negligible side-effects and allows for longterm transgene expression in animal models for delivery of transgenes into the liver, skeletal muscle, myocardium or brain [14]. Furthermore, it does not integrate into the host genome, which makes them a promising class of potential delivery vehicles for human gene therapy [15].

In this study, we developed a HDAd vector containing the full-length JFH1 genome and an HDV ribozyme sequence located at the 3 ' end of the JFH1 genome. Our results demonstrate that the HDAd vector was able to efficiently deliver the HCV genome into Huh-7 cells and HepG2 cells, in which infectious HCV particles could be produced in vitro. To our knowledge, an efficient HDAd-based method for introducing the full-length 9.6 kb HCV RNA genome into hepatocytes has not been previously described. Therefore, this is the first report of an in vitro HDAd-mediated HCV genomic replication and production system.

\section{Results}

Construction of a helper-dependent adenoviral vector expressing the HCV RNA genome

An Ad5 vector has been previously used for the introduction of the hepatitis $\mathrm{B}$ viral genome into cultured cells and mice, and it was found that high-titer hepatitis $B$ virions were secreted into the culture medium of infected hepatoma cells and the sera of infected mice [16]. However, the size of the transgene that can be delivered by the conventional Ad5 vector is limited to 8.1$8.2 \mathrm{~kb}$ [17], which is nearly the size of the HCV replicon. Although an Ad5/35 chimera vector was recently used to generate a HCV subgenomic-replicon construct [18], there have been no reports of the packaging and transfer of the full $9.6 \mathrm{~kb} \mathrm{HCV}$ genome in vitro or in vivo. In this study, we aimed to develop a simple and reliable method for packaging and delivering the HCV genome into cells to establish a novel HCV infection system. To this end, we chose to utilize the genotype $2 \mathrm{a}$ JFH1 HCV clone due to its efficient replication in human hepatoma [19] and mouse cells [20]. The full-length JFH1 RNA genome is under the control of a $5^{\prime}$ minimal CMV promoter in the HDAd vector, and the 3' terminus of the transcript is processed by an HDV ribozyme sequence. The helperdependent adenovirus plasmid also contained a GFP expression cassette (Figure 1a).

Amplification of the HDAdHCV virus and infection of cells As quantified by absorbance, the titer of the amplified and purified HDAdJFH1, HDAdJFH1/GND, HDAdmiR122 and HDAdGFP virus were found to be $2 \times 10^{11}$, $3 \times 10^{11}, 1 \times 10^{11}, 2 \times 10^{11}$ viral particles $/ \mathrm{ml}$, respectively. Huh-7 cells and HepG2 cells were infected by the HDAds at a ratio of 200 viral particles/cell. GFP expression was observed in the infected Huh-7 and HepG2 cells at 3 days p.i. (Figure 1b). At 3 days p.i, nearly all the Huh-7 and HepG2 cells were infected with the HDAdJFH1 virus and had high levels of GFP expression.

\section{Detection of HDAd-mediated HCV protein expression and RNA replication}

HDAd can mediate the efficient delivery and strong expression of foreign DNA sequences in vitro [21]. Our results indicate that HDAd can also mediate the efficient expression of $\mathrm{HCV}$ proteins in HDAdJFH1-infected Huh-7 cells. The expression of HCV core and NS3 proteins was confirmed by immunofluorescence staining and western blotting using core- and NS3-specific monoclonal antibodies. At 3 days p.i., immunofluorescence staining for $\mathrm{HCV}$ core and NS3 were both positive (Figure 2a). High levels of HCV core and NS3 proteins were also detected by western blotting at $36 \mathrm{~h}$ and $72 \mathrm{~h}$ p.i., respectively (Figure $2 \mathrm{~b}$ ). The expression of $\mathrm{HCV}$ NS3 protein was still detectable at 20 days p.i. (data not shown), demonstrating the long-term expression of the $\mathrm{HCV}$ genome.

Actinomycin-D has been shown to have the ability to inhibit transcription [22]. Thus, actinomycin-D was added in the cell culture medium and northern blot was 


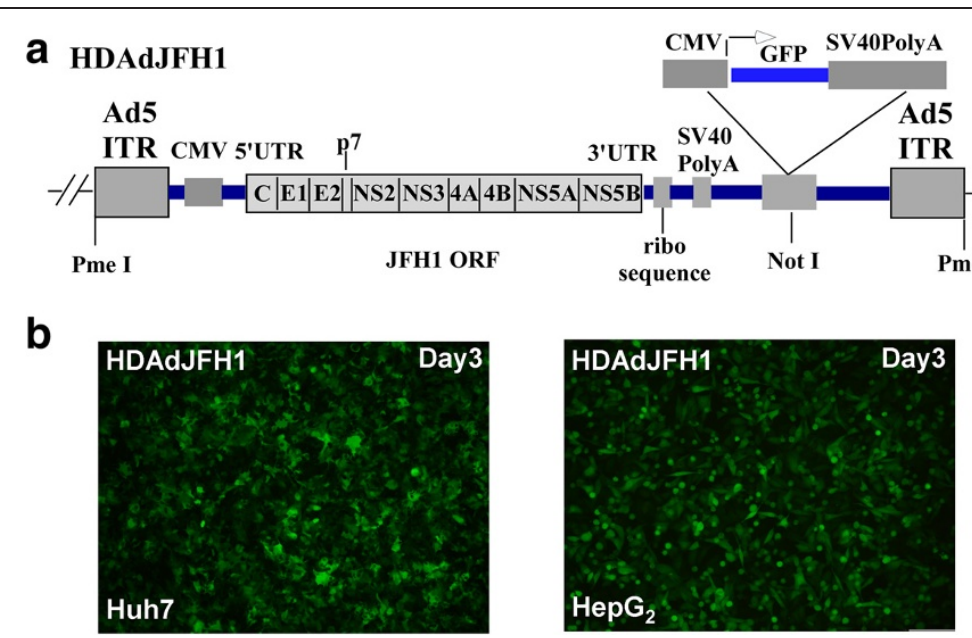

Figure 1 Infection of Huh-7 cells and HepG2 cells by the HDAdJFH1 virus. (a). A diagram depicting the genetic makeup of HDAdJFH1. The transcriptional expression of the JFH1 RNA genome is under the control of the CMV promoter and the HDV ribozyme. The GFP expression cassette is under the control of a second CMV promoter and contains an SV40 polyA tail. (b). GFP expression in HDAdJFH1-infected Huh-7 cells and HepG2 cells on day 3 p.i. Magnification, $\times 10$ (b).

performed to confirm replication of HCV genome. The concentration of actinomycin-D was maintained $5 \mu \mathrm{g} / \mathrm{ml}$ in the medium for 16 hours in HDAdJFH1, HDAdJFH1/ GND-infected Huh-7cells and HDAdJFH1-infected 293 cells since 6 hours p.i. After actinomycin-D treatment, expression of intracellular HCV RNA can be detected in the HDAdJFH1-infected Huh-7 cells, compared with those in the HDAdJFH1/GND-infected Huh-7 cells and HDAdJFH1-infected 293 cells (Figure 2c). The results indicated that actinomycin-D inhibited the HDAdmediated HCV genome transcription in HDAdJFH1/ GND-infected Huh-7 cells and HCV replicated in the HDAdJFH1-infected Huh-7 cells. Quantitative RT-PCR analysis also showed an increase of HCV RNA levels in the cell culture medium since HDAdJFH1 infection of Huh-7 cells (Figure 3). Together, these results demonstrated the replication of HCV genome in the HDAdJFH1-infected Huh-7 cells.
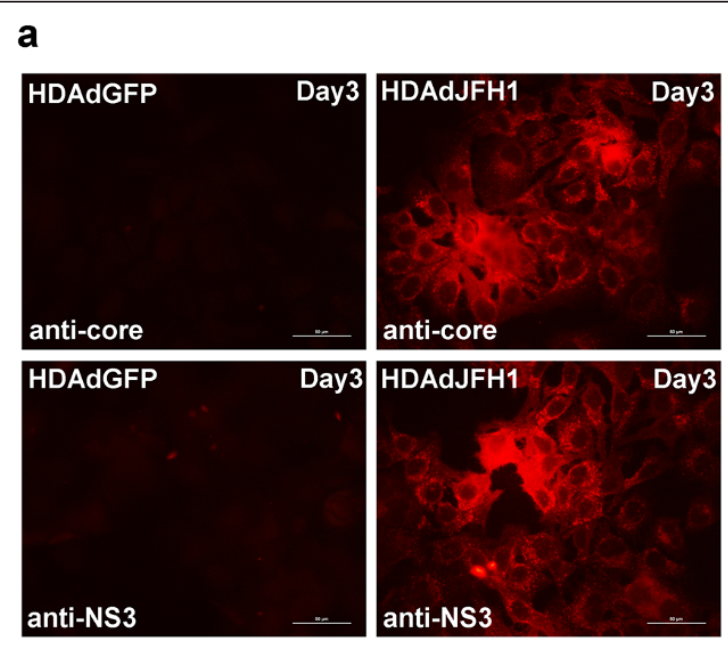

b

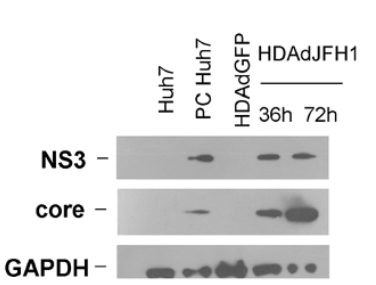

c

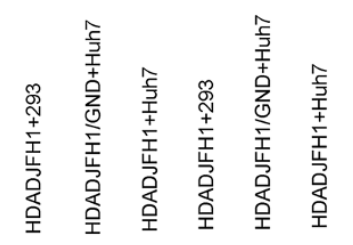

HCV RNA

Actinomycin-D:

Figure 2 HCV expression and replication in HDAdJFH1-infected Huh-7 cells. (a). Immunofluorescence assays (IFAs) of the HCV core and NS3 proteins in Huh-7 cells on day 3 p.i. HDAdGFP-infected Huh-7 cells served as a negative control. (b). Western blots detecting the HCV core and NS3 proteins in HDAdJFH1-infected Huh-7 cells at $36 \mathrm{~h}$ and $72 \mathrm{~h}$ p.i. Huh-7 cells transfected with in vitro-transcribed JFH1 RNA served as a positive control. HDAdGFP-infected Huh-7 cells served as a negative control. (c). Northern blots detecting HCV RNA and 28S rRNA in HDAdJFH1infected Huh-7 cells, HDAdJFH1/GND-infected Huh-7 cells and the HDAdJFH1-infected 293 cells in the presence of actinomycin-D. A group of infected cells without actinomycin-D served as controls. Magnification, $\times 40$ (a). 

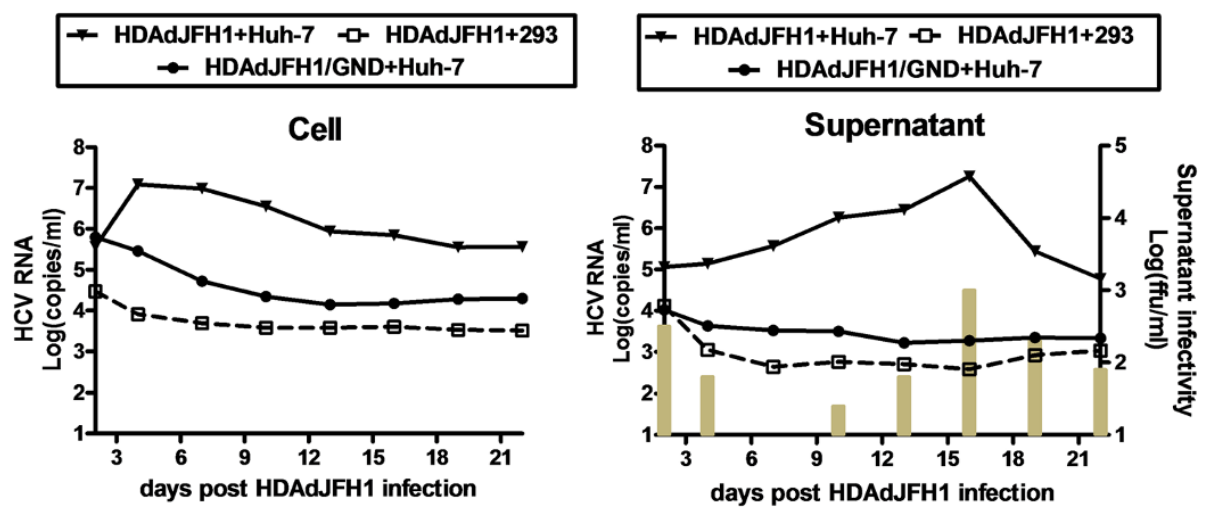

Figure 3 Quantification of the HCV RNA levels in the cells and supernatants. HCV RNA levels were analyzed by RT-qPCR with the HDAdJFH1/GND-infected Huh-7 cells and the HDAdJFH1-infected 293 cells as controls. The supernatant infectivity titers were determined in naïve Huh-7 cells and were expressed as ffu/ml (indicated with bars).

Determination of the infectivity of HDAdJFH1-infected Huh-7 cell culture medium

We found that HCV RNA levels in the cell culture medium of the HDAdJFH1-infected Huh-7 cells first increased to a maximal level at 16 days p.i. and then decreased in the following days. While HCV RNA levels in the supernatant of the HDAdJFH1/GND-infected Huh-7 cells decreased gradually (Figure 3). Based on these findings, we wondered whether HDAd-mediated HCV virions were present in the cell culture medium. To
HDAdJFH1-infected Huh-7 cells, we infected naïve Huh-7 cells with the medium for $1 \mathrm{~h}$ at $37^{\circ} \mathrm{C}$; we subsequently determined the expression levels of the $\mathrm{HCV}$ NS3 protein using IFA. As shown in Figure 4a, naïve Huh-7 cells were infected with medium collected at day 17 p.i., cultured for another 3 days and then assayed for HCV NS3 protein expression using IFA. Naïve Huh-7 cells served as a negative control. Huh-7 cells were also infected with medium from HDAdJFH1/GND-infected Huh-7 cells to confirm that HDAdJFH1 infection
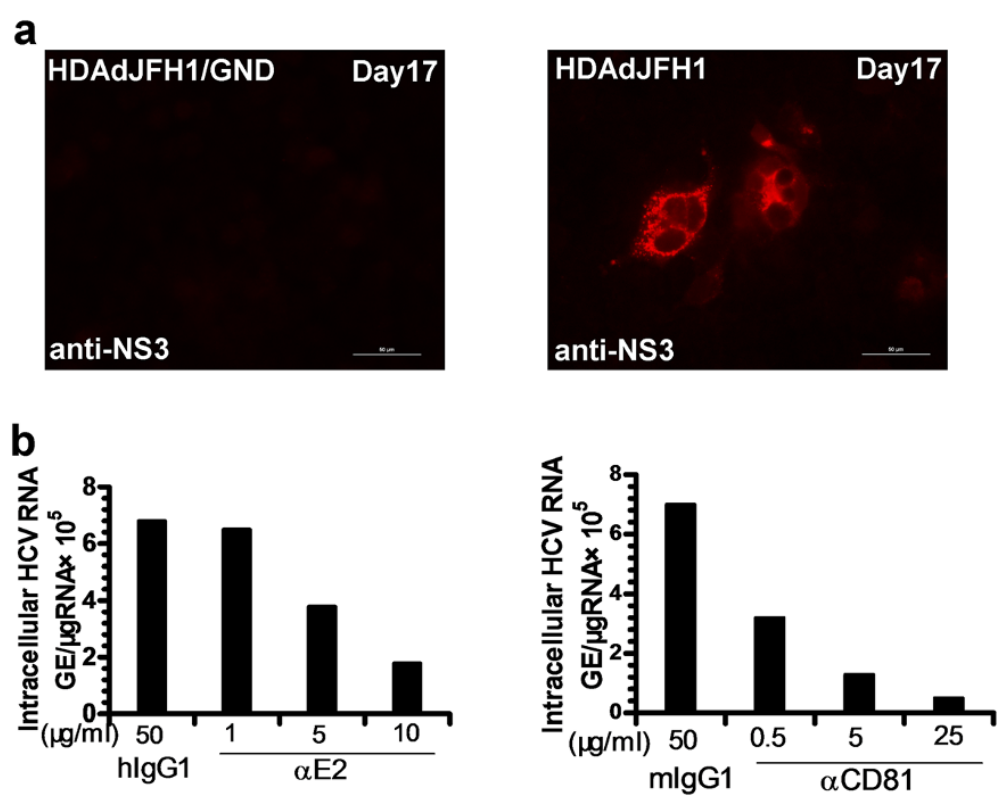

Figure 4 Infectious HCV production in Huh-7 cells following HDAdJFH1 infection. (a). IFA of the HCV NS3 protein in Huh-7 cells infected with supernatant from HDAdJFH1-infected Huh-7 cells that were collected on day 17 p.i. Huh-7 cells infected with supernatant from HDAdJFH1/ GND-infected Huh-7 cells collected on day 17 p.i. served as negative controls. (b). Inhibition of HCV infection by the anti-E2 and anti-CD81 antibody. The infectious HCV supernatants were pre-incubated with the indicated concentrations of anti-E2 or control mouse lgG antibodies for 1 $\mathrm{h}$ at $37^{\circ} \mathrm{C}$ before addition to naïve Huh-7 cells. Huh-7 cells were pre-incubated with the indicated concentrations of anti-human CD81 or control mouse IgG antibodies for $1 \mathrm{~h}$ at $37^{\circ} \mathrm{C}$ before addition of the infectious HCV supernatant. Total cellular RNA was analyzed by RT-qPCR on day 3 p.i. 
mediated HCV replication and infectious HCV secretion into the medium of HDAdJFH1-infected Huh-7 cells.

To determine in more detail the infectious titer of $\mathrm{HCV}$, the medium was serially diluted in 10-fold increments and used to infect Huh-7 cells. The infectious titer was determined by counting the number of cell foci that stained positive for HCV NS3 protein at the lowest dilution point, which was then multiplied by the dilution factor (n-fold) [6]. The infectious $\mathrm{HCV}$ titer of the medium reached a maximal level of $1 \times 10^{3} \mathrm{ffu} / \mathrm{ml}$ at 16 days p.i. (Figure 3) and was comparable to the infectious HCV titer in Huh-7 cell by conventional transfection of in vitro transcribed JFH1 RNA, which normally only reaches $10^{2} \sim 10^{3} \mathrm{ffu} / \mathrm{ml}$.

\section{HCV infection is inhibited by anti-E2 and anti-CD81 antibodies}

Previous studies using pseudotyped viruses expressing HCV E1/E2 have suggested that the interaction between E2 and CD81 is crucial for viral entry [23,24]. To further investigate the infectivity of the $\mathrm{HCV}$ virions, we performed infectivity-neutralization and inhibition experiments using monoclonal antibodies specific for $\mathrm{HCV}$ E2 and CD81. Prior to addition to the Huh-7 cells, the infectious $\mathrm{HCV}$ supernatant was pre-incubated with a dilution series of a mouse monoclonal antibody specific for HCV E2. An irrelevant human IgG1 antibody served as a negative control. In another experiment, the Huh-7 cells were pre-incubated with CD81 antibodies before the infectious HCV supernatant was added. The irrelevant mouse IgG1 antibody served as a negative control. For both the E2 and CD81 antibody treatments, the intracellular HCV RNA levels were observed to decrease in a dose-dependent manner by 3 days p.i. A concentration of $5 \mu \mathrm{g} / \mathrm{ml}$ of anti-E2 antibody was sufficient to cause a $50 \%$ reduction in the intracellular HCV RNA level by 3 days p.i., whereas only $0.5 \mu \mathrm{g} / \mathrm{ml}$ of anti-CD 81 antibody was sufficient to cause the same reduction
(Figure 4b). These results are consistent with the previously reported findings [6,7] and confirm the infectivity of the cell culture medium.

To characterize the produced HCV virions, the culture medium of the HDAdJFH1-infected Huh-7 cells was subjected to sucrose density gradient centrifugation. The fractions were analyzed for HCV RNA levels, as well as the infectivity titers. As shown in Figure 5a, a peak for HCV RNA level and infectivity titer was found in fraction 4 , which has a density of $1.15 \mathrm{~g} / \mathrm{ml}$. This density is almost consistent with the published density of HCV virions $[8,25]$. The infection kinetics assay showed that the cellular HCV RNA levels increased to $10^{7}$ copies within 7 days p.i. (Figure 5b). These results further confirm the production of infectious $\mathrm{HCV}$ virions in this HDAdmediated system.

\section{Inhibition of HCV replication by IFN-a}

Previous studies have shown that IFN- $\alpha$ can successfully inhibit HCV replication both in Huh-7 cells transfected with JFH1 RNA [6] and in Huh-7 cells with a stably integrated HCV genome [8]. Therefore, we investigated the inhibitory effects of IFN- $\alpha$ on HCV replication in our HDAd-mediated infection system. Beginning at 8 days p.i., HDAdJFH1-infected Huh-7 cells were incubated with DMEM containing different concentrations of IFN$\alpha$ for 3 days, and levels of the NS3 protein were determined by western blotting. IFN- $\alpha$ efficiently inhibited $\mathrm{HCV}$ replication in HDAdJFH1-infected Huh-7 cells (Figure 6). Therefore, it is likely that this HDAdmediated HCV infection system is suitable for the evaluation of new anti-HCV drugs and therapeutic strategies.

HDAdJFH1 and HDAdmiR-122 virus co-infection mediate HCV expression and replication in HepG2 cells

HepG2 cells does not express endogenous miR-122, a liver-expressed miRNA which is required to support HCV RNA replication [10], and weakly supports HCV
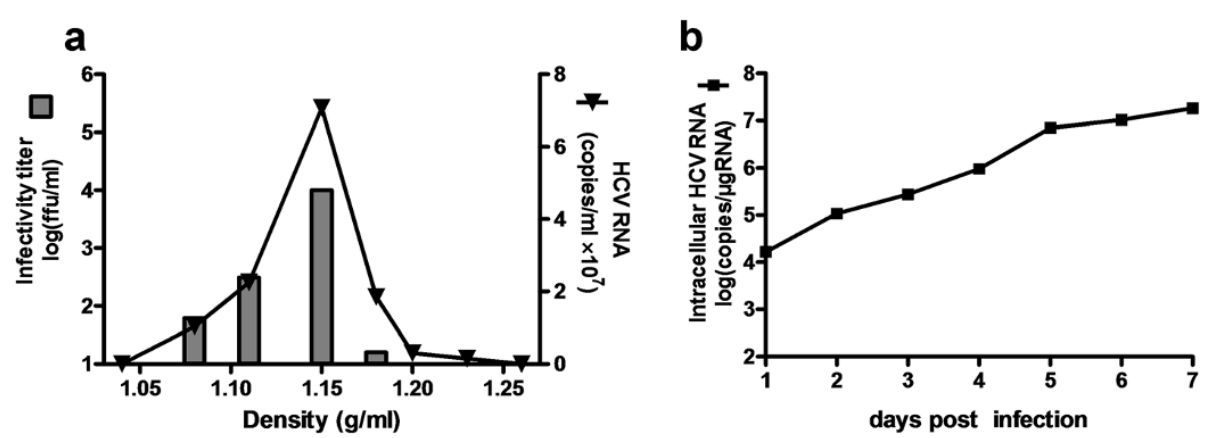

Figure $\mathbf{5}$ HCV infectivity determination by density gradient analysis and infection kinetics assay. (a). Density gradient analysis of produced HCV particles. Concentrated culture medium collected from HDAdJFH1-infected Huh-7 cells was fractionated, HCV RNA levels and HCV infectivity titers in each fraction was determined. (b). Infection of naïve Huh-7 cell by the supernatant from the HDAd-JFH1-infected Huh-7 cells and infection kinetics assay analysis. 


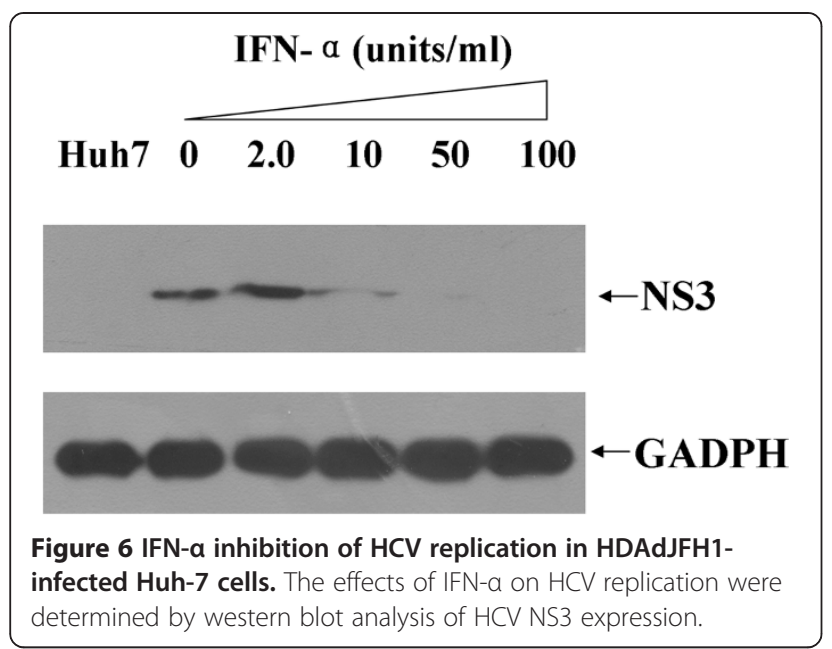

replication [11]. A recent study has indicated that HepG2 cells expressing miR-122 can support the entire HCV life cycle [11]. In this study, we tested whether the HDAd system can work in HepG2 cells. Results showed that co-infection of HepG2 cells with HDAdJFH1 and HDAdmiR-122 virus enhances HCV core and NS3 protein expression (Figure 7a). Kinetic analysis of $\mathrm{HCV}$ RNA levels in the co-infected HepG2 cells and culture medium were performed. Compared with the HDAdJFH1/ GND infection control, HDAdJFH1 and HDAdmiR-122 co-infection resulted in relatively weak replication of $\mathrm{HCV}$ genome, as shown in Figure 7b.

\section{Discussion}

Hepatitis $\mathrm{C}$ virus (HCV) is a positive-strand RNA virus that was first identified in 1989. Since then, both basic and pharmaceutical research on $\mathrm{HCV}$ has been hampered by a lack of viral culturing systems [4]. Early studies on HCV mainly focused on the structure and function of the individual viral genes, and later studies utilized HCV subgenomic replicons, which were more effective for HCV RNA replication research and antiviral development $[20,26]$. However, the replicon system is not suitable for HCV life-cycle research due to this system's inability to produce infectious $\mathrm{HCV}$ virions. In 2005, it was reported that RNA generated from the JFH1 viral genome can produce infectious $\mathrm{HCV}$ virions when transfected into Huh-7 cells [7]. Both transient and stable transfections with DNA vectors expressing the




full-length $\mathrm{HCV}$ genome have also been investigated $[8,25]$ and in these studies it was found that the HDV ribozyme plays an important role in cleavage of the HCV RNA product.

It appears that efficient delivery of the HCV RNA genome, for example by electroporation or stable transfection, is vital for the efficient replication and production of the HCV virus. Here, we report on the use of the helper-dependent adenoviral (HDAd) vector, which can mediate high-efficiency transduction of one or more foreign genes into cells [27]. Adenoviruses (Ads) are nonenveloped double-stranded DNA viruses, replication and assembly of which take place in the nucleus. Adenovirus vectors are most effective at mediating the transfer of foreign DNA into immortalized and primary cells. In the liver, they predominantly infect hepatocytes [21], where hepatitis-virus replication and infection take place. Adenoviral vectors have been used for the transfer of the genome of the hepatitis B virus into cultured cells and mice [16]. However, the $9.6 \mathrm{~kb}$ HCV RNA genome was too large to be introduced into early generation Ad vectors. HDAds are constructed by removing all viral sequences from earlier generation Ad vectors except for the packaging sequences and the inverted terminal repeats, which eliminates viral gene expression, liver toxicity, and the cellular immune responses elicited by Ad vectors [28]. As most of the viral coding sequences are deleted, HDAds have a large delivery capacity of up to $37 \mathrm{~kb}$ [29]. As a comparison, the upper size limit of transgenes that can be delivered with the conventional Ad5 vector is 8.1-8.2 kb [17] - approximately the size of the HCV replicon. Thus, it has been difficult to package the $\mathrm{HCV}$ replicon into Ad5, although an Ad5/35 chimera vector has recently been used to generate an HCV subgenomic-replicon construct [11]. To our knowledge, a convenient and efficient method for transferring the $9.6 \mathrm{~kb}$ HCV RNA genome into cells by HDAds has not been previously reported.

In this study, we developed an HDAd vector containing the full-length JFH1 genome and an HDV ribozyme sequence. HDAdJFH1 virus with a high infectious titer was prepared and used to infect Huh-7 cells in vitro, and we observed both long-term expression and replication of HCV genome (Figures 2 and 3). HCV replication was confirmed by de novo RNA synthesis in the presence of actinomycin-D. The HCV RNA levels in the supernatants increased to a maximal level of about $10^{7}$ copies/ml at 16 days p.i. when many cells became rounded and floated in the culture medium, a similar cytopathic effect that can be observed on the JFH1transfected Huh-7 cells [30]. Ten days after infection, the supernatant from HDAdJFH1-infected Huh-7 cells was found to be infectious and the infectivity of $\mathrm{HCV}$ could be inhibited by anti-E2 and anti-CD 81 antibodies in a dose-dependent manner (Figure 4b). As the interaction between E2 and CD81 is vital for HCV entry $[31,32]$, we believed that HCV particles were being secreted into the supernatant of HDAdJFH1-infected Huh-7 cells. Sucrose density gradient analysis showed that the virus produced owns a density which is similar to published data for $\mathrm{HCV}$ virions [25]. Furthermore, we found that IFN- $\alpha$ efficiently inhibited HCV replication (Figure 6), further validating the potential of this system for the evaluation of HCV antiviral drugs. Taken together, our findings demonstrate long-term replication of the HCV genome and production of infectious $\mathrm{HCV}$ virions in this helper-dependent adenovirusmediated system in Huh-7 cells.

MicroRNAs (miRNA) are 21 22 nucleotide RNA molecules that are expressed in a wide range of eukaryotic organisms that are predicted to downregulate the expression of the endogenous target genes by reducing mRNA stability or translation [33]. MiR-122 is liverspecific and required for efficient HCV RNA replication $[10,34]$. HepG2 cells does not express endogenous miR122 and weakly supports HCV replication [11]. A recent study has indicated that HepG2 cells expressing miR-122 can support the entire HCV life cycle [11]. In this study, our results also showed that HDAd-mediated miR-122 expression was necessary for the HDAd-mediated efficient HCV expression and replication (Figure 7).

Although the method based on in vitro-transcribed JFH1-RNA transfection is very well established so far, it is less efficient for primary hepatocytes which are difficult to transfect. Helper-dependent adenovirus can infect a variety of different cell types, including hepatic cell lines, primary hepatocytes [35], as well as quiescent, non-dividing, or terminally differentiated somatic cells [36], with high expression efficiency. Thus, by using this HDAd system, HCV replication or infection can be studied in the above cell types in vitro. So far, HCV can only infect human and chimpanzees in vivo and the host range for $\mathrm{HCV}$ infection is narrow. Although human liver chimeric mice can be infected by HCV, it is immunodeficient with a high mortality rate and the source of human primary hepatocytes is limited. And it is important to generate a novel immunocompetent mouse model which can support $\mathrm{HCV}$ replication and infection. Although expression of the four humanized HCV receptors in mice enhance its susceptibility to HCV infection $[37,38]$, it is still not successful for mediating HCV infection in mice. Several studies have shown that HDAds are able to mediate high-level and long-term transgene expression in animal models, especially for the livertargeted gene therapy $[13,14]$. So it is probable that HDAd can mediate efficient delivery of the HCV genome into the mouse livers, but whether HDAd can mediate $\mathrm{HCV}$ genomic replication or even virion 
production in mice is worth being investigated. Also, since extrahepatic manifestations caused by hepatitis $\mathrm{C}$ virus infection exists clinically [39], animal model for studying HCV extrahepatic manifectations still lacks. And this HDAd-mediated HCV genomic replication system provides a possible strategy for generating an $\mathrm{HCV}$ extrahepatic replication model in vivo.

\section{Conclusion}

This is the first report of an in vitro HDAd-mediated $\mathrm{HCV}$ genomic replication and production system, which would allow a broader examination of interactions between $\mathrm{HCV}$ and host cell biology, as well as provide tools for anti-HCV drug evaluation.

\section{Methods}

Helper-dependent adenoviral vector construction

The plasmid pJFH1 contains the full-length JFH1 cDNA downstream of the T7 RNA promoter [7]. To replace the T7 promoter from PJFH1 with CMV promoter, a PCR fragment, amplified 5 ' partial sequence of the $\mathrm{HCV}$ genome in pJFH1 using the chimeric forward primer 5'ccgaattcgagctcggtacccgg-3' which consists of two segments lying beside the upstream and downstream of T7 promoter and reverse primer $5^{\prime}$-caccggttccgcagaccac-3', was inserted into pJFH1 between the restriction enzyme sites for EcoR I and Age I that locates upstream and downstream of the T7 promoter respectively. The resulting construct was designated $\mathrm{pJFH} 1(\Delta \mathrm{T} 7)$. A hepatitis delta virus (HDV) ribozyme DNA sequence was synthesized and placed at the immediate $3^{\prime}$ end of pJFH1( $\Delta \mathrm{T} 7)$, to get pJFH1-ribo( $\Delta \mathrm{T} 7)$. The JFH1-ribo sequence was then inserted between the CMV promoter and SV40PolyA of the pSC11 plasmid by the restriction enzyme sites for EcoR I and Hind III. And this expression cassette was finally inserted into the HDAd vector between the restriction enzyme sites for I-Ceu I and ISce I. The resulting construct, HDAdJFH1, also contains a cassette for the expression of green fluorescent protein (GFP) [40]. HDAdJFH1/GND was constructed with the same strategy. And an empty HDAd plasmid expressing GFP (HDAdGFP) served as a control.

Using the Huh-7 cells derived total RNA-reverse transcribed cDNA as a template, the human genomic miR-122 precursor sequence was amplified by RT-PCR using the forward and reverse primers 5 '-ccggaattcttcgtggctacagagttt- 3 ' and $5^{\prime}$-cccaagcttttatcgagggaaggatt- 3 ', and then inserted into the pSC11 plasmid by the restriction enzyme sites for EcoR I and Hind III. And this expression cassette was finally inserted into the HDAd vector between the restriction enzyme sites for $I-\mathrm{Ceu} \mathrm{I}$ and $\mathrm{I}$-Sce I. The resulting construct was designated HDAdmiR-122.

\section{Cells and cell culture methods used in this study}

A modified version of the 293 cell line that expresses high levels of the Cre enzyme - known as the 116 cell line (kindly provided by Dr. Li-min Liu) - was maintained in MEM supplemented with $10 \%$ fetal bovine serum (FBS), $0.1 \mathrm{mg} / \mathrm{ml}$ hygromycin, $100 \mathrm{U} / \mathrm{ml}$ penicillin, $100 \mu \mathrm{g} / \mathrm{ml}$ streptomycin, and $2 \mathrm{mM}$ L-glutamine (Invitrogen) [41]. The hepatoma cell lines Huh-7, HepG2, as well as nonhepatic 293 cell line, were obtained from ATCC (Manassas, VA) and maintained in DMEM supplemented with $100 \mathrm{U} / \mathrm{ml}$ penicillin, $100 \mu \mathrm{g} / \mathrm{ml}$ streptomycin, nonessential amino acids, and 10\% FBS (Invitrogen).

\section{Amplification of the HDAdHCV virus and infection of cells}

The helper-dependent adenoviral plasmids, including HDAdJFH1, HDAdJFH1/GND, HDAdmiR-122, HDAdGFP, were digested with the restriction enzyme PmeI to release both inverted terminal repeats and were then transfected into 116 cells with the calcium phosphate method, respectively [42]. Helper-dependent adenoviral particles were prepared by repeated amplification cycles in 116 cells with the helper virus AdNG163 (a kind gift of Dr. Li-min Liu; see above). After 4-6 rounds of amplification in 150-mm dishes, 116 cells suspended in a $3 \mathrm{~L}$ spinner flask were infected for large-scale preparation of HDAdJFH1, HDAdJFH1/GND, HDAdmiR-122, HDAdGFP. The viral particles were isolated by centrifugation in a cesium chloride gradient, followed by dialysis with three exchanges of 10 $\mathrm{mM}$ Tris- $\mathrm{HCl}(\mathrm{pH} 8.0)$ at $4^{\circ} \mathrm{C}$ [43]. Virus particles were quantified by measuring the absorbance at $260 \mathrm{~nm}$ [44]. Huh-7, $\mathrm{HepG}_{2}$ and 293 cells were infected with the purified HDAds at $80 \%$ confluency with an MOI of 200 viral particles/cell and were passaged every 2 or 3 days.

\section{Western blot analysis}

The HDAdJFH1-infected Huh-7 cells were lysed in protein sample buffer containing $50 \mathrm{mM}$ Tris- $\mathrm{HCl}, 150 \mathrm{mM}$ $\mathrm{NaCl}, 5 \mathrm{mM}$ EDTA, $0.2 \mathrm{mM}$ sodium orthovanadate, $1 \%$ Triton X-100, 1\% sodium deoxycholate, and 1\% sodium dodecyl sulfate; the buffer was also supplemented with aprotinin $(2 \mu \mathrm{g} / \mathrm{ml})$, pepstatin A $(0.7 \mu \mathrm{g} / \mathrm{ml})$, leupeptin $(0.5 \mu \mathrm{g} / \mathrm{ml})$, and PMSF $(1 \mathrm{mM})$. For each sample, $30 \mu \mathrm{g}$ of total protein was electrophoresed through a $10 \%$ sodium dodecyl sulfate-polyacrylamide gel and transferred onto a nitrocellulose membrane. The membrane was blocked by pre-incubation with $5 \%$ skim milk. The levels of the $\mathrm{HCV}$ core and NS3 proteins were determined using monoclonal antibodies specific to the core and NS3 proteins (Thermo Scientific), which were detected with a horseradish peroxidase-conjugated goat anti-mouse immunoglobulin G antibody (IgG, Pierce) and visualized with a chemiluminescent substrate (Pierce). The level of the GAPDH protein served as an internal control, and this 
protein was detected with an anti-GAPDH monoclonal antibody (Sigma).

\section{Immunofluorescence assays (IFAs)}

The HDAdJFH1-infected Huh-7 and HepG2 cells were grown overnight on coverslips in a 6-well culture plate. Cells were washed with $1 \times$ phosphate-buffered saline (PBS), fixed with chilled acetone, and blocked with $1 \%$ bovine serum albumin and $1 \%$ goat serum in PBS. The localizations of the $\mathrm{HCV}$ core and NS3 proteins were visualized in the fixed cells by incubation with core- and NS3-specific monoclonal antibodies and a secondary goat anti-mouse IgG antibody conjugated to DyLight ${ }^{\mathrm{m}} 594$ fluorescein (used at a 1:800 dilution) (Beijing Golden Bridge Biotechnology, Beijing, China). The HDAdGFPinfected Huh-7 and HepG2 cells served as a negative control. The coverslips were then mounted onto slides, and the HCV proteins were visualized with a Zeiss Axioplan 2 fluorescence microscope.

\section{Quantification of HCV RNA levels}

Total cellular RNA was extracted from infected cells using the RNeasy RNA isolation kit (Qiagen). Total RNA was extracted from the supernatants of the infected cells using the QIAamp Viral RNA Mini Kit (Qiagen). The RNA concentration was determined by spectrophotometry. RT-qPCR was performed as described elsewhere [6], and the HCV levels were determined relative to a standard curve created using serial dilutions of a plasmid containing the HCV JFH1 cDNA.

\section{Northern blot analysis of intracellular viral RNA levels}

To confirm the replication of HCV genome, actinomycin$\mathrm{D}$ (Sigma) was maintained in the cell culture medium at a concentration of $5 \mu \mathrm{g} / \mathrm{ml}$ for 16h in HDAdJFH1, HDAdJFH1/GND-infected Huh-7cells and HDAdJFH1infected 293 cells since 6 hours p.i.. Total cellular RNA was extracted from infected Huh-7 cells using the RNeasy RNA isolation kit (Qiagen). Four micrograms of isolated RNA was separated using a 1\% agarose gel containing formaldehyde, blotted onto a positively charged nylon membrane (Hybond-N+, GE), and immobilized with a Stratalinker UV crosslinker (Stratagene). The DNA probes complementary to a region of the HCV 5'UTR and human 28S rRNA was synthesized using the DIG High Prime DNA Labeling and Detection Starter Kit II (Roche).

\section{$\mathrm{HCV}$ infection and infectivity neutralization}

Naïve Huh-7 cells in a 6-well culture plate were infected with $1 \mathrm{ml}$ of cultured supernatant from HDAdJFH1infected Huh-7 cells. At $3 \mathrm{~h}$ postinfection (p.i.), the supernatants were replaced with $2 \mathrm{ml}$ of DMEM containing $10 \% \mathrm{FBS}$, and the cells were incubated at $37^{\circ} \mathrm{C}$ for 3 days prior to the protein and RNA analysis. At 3 days p.i., the
$\mathrm{HCV}$ infectivity was determined by IFA for the HCV core and NS3 proteins using core- and NS3-specific monoclonal antibodies, respectively. Titration of infectious $\mathrm{HCV}$ was performed by focus forming assay. Cell supernatants were serially diluted 10-fold in complete DMEM and used to infect naïve Huh-7 cells. The level of infectious $\mathrm{HCV}$ titer was determined by the average number of NS3positive foci detected at highest dilutions at 3 days p.i.. For the infectivity-neutralization experiments, monoclonal antibodies specific to HCV E2 and CD81 (Santa Cruz Biotechnology) were diluted with the $\mathrm{HCV}$-containing culture medium at 3 days p.i.; normal mouse IgG1 (Santa Cruz Biotechnology) antibodies were used as negative controls. The effects of the E2 and CD81 monoclonal antibodies on HCV infectivity and replication were determined by RTqPCR.

\section{Sucrose density gradient analysis}

We collected the cell culture medium of the HDAdJFH1infected Huh-7 cells at 16 days p.i. using low-speed centrifugation and passed it through a $0.45-\mu \mathrm{m}$ filter. Then the filtrate was layered on a sucrose gradient $(60 \% \sim 10 \%$, wt/vol) and centrifuged it for $16 \mathrm{~h}$. The fractions were harvested and analyzed for the HCV RNA levels and infectivity titers.

\section{IFN- $a$ inhibition of HCV replication in HDAdJFH1-infected Huh-7 cells}

For the IFN- $\alpha$ inhibition experiments, Huh-7 cells were infected with HDAdJFH1 and incubated with DMEM containing increasing concentrations of IFN- $\alpha$ (Sigma) for 3 days. The effects of IFN- $\alpha$ on HCV replication were determined using western blot analysis of HCV NS3 expression.

\section{Abbreviations}

HCV: Hepatitis C virus; CDNA: Complementary DNA; HCVcc: infectious HCV in cell culture; HDAd: Helper-dependent adenovirus; ml: milliliter; IFN-a: Interferona; RNA: Rinonucleic acid; ER: Endoplasmic reticulum; UTR: Untranslated region; ORF: Open reading frame; miRNA: microRNA; IFA: Immunofluorescence assay; RT-PCR: Reverse transcription-polymerase chain reaction; ffu: foci forming unit; p.i.: postinfection; DMEM: Dulbecco's Modified Eagle Medium;

CMV: Cytomegalovirus; FBS: Fetal bovine serum; ATCC: American type culture collection; MOI: Multiplicity of infection; EDTA: Ethylenediaminetetraacetic acid; PMSF: Phenylmethanesulfonyl fluoride; RT-qPCR: Real-time quantitative polymerase chain reaction; DIG: Digoxigenin.

\section{Competing interests}

The authors have declared that no competing interests exists.

\section{Authors' contributions}

Conceived and designed the experiments: $Y Z$ and S-hS. Performed the experiments: $X-j Z, Y Z, J L, Y G$, and $Y-h F$. Analyzed the data: $X-j Z$ and $Y Z$. Wrote and revised the manuscript: $X-j Z, J-s H$, and $Y$-sZ. All authors read and approved the final manuscript.

\section{Acknowledgments}

We are grateful to Professor Yue Wang (Chinese Center for Disease Control and Prevention) for providing the pJFH1 and pJFH1/GND plasmid, to Dr. Li-min Liu (McMaster University) for providing the Cre-expressing 293 cell line and the HDAd vector, and to Professor Jing-sheng He (Beijing Jiaotong University) for assistance in production of HDAdJFH1 virus. 


\section{Funding}

This work was supported by National Program of Infectious Diseases (No.2012ZX10004-502) and The National High Technology Research and Development Program ("863" Program) of China (No.2007AA02Z151).

\section{Author details}

${ }^{1}$ State Key Laboratory of Pathogen and Biosecurity, Beijing Institute of Microbiology and Epidemiology, Beijing, China. ${ }^{2}$ Laboratory Animal Center of the Academy of Military Medical Science, Beijing, China. ${ }^{3}$ College of Life Sciences \& Bioengineering, Beijing Jiaotong University, Beijing, China.

Received: 28 December 2012 Accepted: 26 August 2013

Published: 30 August 2013

\section{References}

1. Alter MJ, Margolis HS, Krawczynski K, Judson FN, Mares A, Alexander WJ, Hu PY, Miller JK, Gerber MA, Sampliner RE, The Sentinel Counties Chronic non-A, nonB Hepatitis Study Team: The natural history of community-acquired hepatitis C in the United States. N Engl J Med 1992, 327:1899-1905.

2. Sarbah SA, Younossi ZM: Hepatitis C: an update on the silent epidemic. J Clin Gastroenterol 2000, 30:125-143.

3. Hoofnagle $\mathrm{JH}$, di Bisceglie AM: The treatment of chronic viral hepatitis. N Engl J Med 1997, 336:347-356.

4. Reed KE, Rice CM: Overview of hepatitis C virus genome structure, polyprotein processing, and protein properties. Curr Top Microbiol Immunol 2000, 242:55-84.

5. Lohmann V, Korner F, Koch JO, Herian U, Theilmann L, Bartenschlager R: Replication of subgenomic hepatitis C virus RNAs in a hepatoma cell lines. Science 1999, 285:110-113.

6. Zhong J, Gastaminza P, Cheng G, Kapadia S, Kato T, Burton DR, Wieland SF, Uprichard SL, Wakita T, Chisari FV: Robust hepatitis C virus infection in vitro. Proc Natl Acad Sci USA 2005, 102:9294-9299.

7. Wakita T, Pietschmann T, Kato T, Date T, Miyamoto M, Zhao Z, Murthy K, Habermann A, Krausslich HG, Mizokami M, Bartenschlager R, Liang TJ: Production of infectious hepatitis $C$ virus in tissue culture from a cloned viral genome. Nat Med 2005, 11:791-796.

8. Cai ZH, Zhang C, Chang KS, Jiang JY, Ahn BC, Wakita T, Liang TJ, Luo GX Robust production of infectious hepatitis C virus (HCV) from stably HCV cDNA-transfected human hepatoma cells. J Virol 2005, 79:13963-13973.

9. Decaens C, Durand M, Grosse B, Cassio D: Which in vitro models could be best used to study hepatocyte polarity? Biol Cell 2008, 100:387-398.

10. Jopling CL, Yi M, Lancaster AM, Lemon SM, Sarnow P: Modulation of hepatitis C virus RNA abundance by a liver-specific MicroRNA. Science 2005, 309:1577-1581.

11. Narbus CM, Israelow B, Sourisseau M, Michta ML, Hopcraft SE, Zeiner GM Evans MJ: HepG2 cells expressing miR-122 support the entire hepatits $C$ virus life cycle. J Virol 2011, 85(22):12087-12092.

12. Davis AR, Wivel NA, Palladino JL, Tao L, Wilson JM: Construction of adenoviral vectors. Mol Biotechnol 2001, 18:63-70.

13. Vetrini F, Philip N: Gene therapy with helper-dependent adenoviral vectors: current advances and future perspectives. Viruses 2010, 2:1886-1917.

14. Jozkowicz A, Dulak J: Helper-dependent adenoviral vectors in experiment gene therapy. Acta Biochim Pol 2005, 52:589-599.

15. Adam E, Nasz I: Adenovirus vectors and their clinical application in gene therapy. Orv Hetil 2001, 142:2061-2070.

16. Sprinzl MF, Oberwinkler H, Schaller H, Protzer U: Transfer of hepatitis B virus genome by adenovirus vectors into cultured cells and mice: crossing the species barrier. J Virol 2001, 75:5108-5118.

17. Bett AJ, Prevec L, Graham FL: Packaging capacity and stability of human adenovirus type 5 vectors. J Virol 1993, 67:5911-5921.

18. Yoshida T, Kondoh M, Ojima M, Mizuguchi H, Yamagishi Y, Sakamoto N, Yagi K: Adenovirus vector-mediated assay system for hepatitis $C$ virus replication. Nucleic Acids Res 2011, 39:e1-8.

19. Kato T, Date T, Miyamoto M, Furusaka A, Tokushige K, Mizokamo M, Wakita T: Efficient replication of the genotype 2a hepatitis $C$ virus subgenomic replicon. Gastroenterology 2003, 125:1808-1817.

20. Uprichard SL, Chung J, Chisari FV, Wakita T: Replication of a hepatitis C virus replicon clone in mouse cells. Virol I 2006, 3:89.

21. Palmer DJ, Ng P: Helper-dependent adenoviral vectors for gene therapy. Hum Gene Ther 2005, 16:1-16.

22. Sobell H: Actinomycin and DNA transcription. Proc Natl Acad Sci USA 1985, 82:5328-5331.
23. Hsu M, Zhang J, Flint M, Logvinoff C, Cheng-Mayer C, Rice CM, McKeating JA: Hepatitis $\mathrm{C}$ virus glycoproteins mediate $\mathrm{pH}$-dependent cell entry of pseudotyped retroviral particles. Proc Natl Acad Sci USA 2003, 100:7271-7276.

24. Zhang J, Randall G, Higginbottom A, Monk P, Rice CM, McKeating JA: CD81 is required for hepatitis $C$ virus glycoprotein-mediated viral infection. J Virol 2004, 78:1448-1455.

25. Heller T, Saito S, Auerbach J, Williams T, Moreen TR, Jazwinski A, Cruz B, Jeurkar N, Sapp R, Luo G, Liang TJ: An in vitro model of hepatitis C virion production. Proc Natl Acad Sci USA 2005, 102:2579-2583.

26. Bartenschlager RA, Kaul SS: Replication of the hepatitis C virus in cell culture. Antiviral Res 2003, 60:91-102.

27. Sullivan DE, Dash S, Du H, Hiramatsu N, Aydin F, Kolls J, Blanchard J, Baskin $\mathrm{G}$, Gerber MA: Liver-directed gene transfer in non-human primates. Hum Gene Ther 1997, 8:1195-1206.

28. Muruve DA, Cotter MJ, Zaiss AK, White LR, Liu Q, Chan T, Clark SA, Ross PJ, Meulenbroek RA, Maelandsmo GM, Parks RJ: Helper-dependent adenovirus vectors elicit intact innate but attenuated adaptive host immune responses in vivo. J Virol 2004, 78:5966-5972.

29. Kochanek S: High-capacity adenoviral vectors for gene transfer and somatic gene therapy. Hum Gene Ther 1999, 10:2451-2459.

30. Zhong J, Gastaminza P, Chung J, Stamataki Z, Isogawa M, Cheng GF, McKeating JA, Chisari FV: Persistent hepatitis C virus infection in vitro: coevolution of virus and host. J Virol 2006, 80:11082-11093.

31. Cormier EG, Tsamis F, Kajumo F, Durso RJ, Gardner JP, Dragic T: CD81 is an entry coreceptor for hepatitis C virus. Proc Natl Acad Sci USA 2004, 101:7270-7274.

32. Pileri $P$, Uematsu $Y$, Campagnoli $S$, Galli G, Falugi F, Petracca R, Weiner AJ, Houghton M, Rosa D, Grandi G, Abrignani S: Binding of hepatitis C virus to CD81. Science 1998, 282:938-942.

33. Ambros V: The fuctions of animal microRNAs. Nature 2004, 431:350-355.

34. Jangra RK, Yi M, Lemon SM: Regulation of hepatitis C virus translation and infectious virus production by the microRNA miR-122. J Virol 2010, 84:6615-6625.

35. Salucci V, Lu M, Aurisicchio L, La Monica N, Roggendorf M, Palombo F: Expression of a new woodchuck IFN-alpha gene by a helper-dependent adenoviral vector in woodchuck hepatitis virus-infected primary hepatocytes. J Interferon Cytokine Res 2002, 22:1027-1034.

36. Kubo S, Seleme MC, Soifer HS, Perez JL, Moran JV, Kazazian HH Jr, Kasahara $\mathrm{N}$ : L1 retrotransposition in nondividing and primary human somatic cells. Proc Natl Acad Sci USA 2006, 103:8036-41.

37. Dorner M, Horwitz JA, Robbins JB, Barry WT, Feng Q, Mu K, Jones CT, Schoggins JW, Catanese MT, Burton DR, Law M, Rice CM, Ploss A: A genetically humanized mouse model for hepatitis $C$ virus infection. Nature 2011, 474:208-211.

38. Hikosaka K, Noritake H, Kimura W, Sultana N, Sharkar MT, Tagawa Y, Uezato T, Kobayashi Y, Wakita T, Miura N: Expression of human factors CD81, claudin-1, scavenger receptor, and occluding in mouse hepatocytes does not confer susceptibility to HCV entry. Biomedical Res 2011, 32:143-150.

39. Zignego AL, Ferri C, Pileri SA, Caini P, Bianchi FB: Extrahepatic manifestations of hepatitis $C$ virus infection: a general overview and guidelines for a clinical approach. Dig Liver Dig 2007, 39:2-17.

40. Shi CX, Graham FL, Hitt MM: A convenient plasmid system for construction of helper-dependent adenoviral vectors and its application for analysis of the breast-cancer-specific mammaglobin promoter. J Gene Med 2006, 8:442-451.

41. Liu L, Shi CX, Ghayur A, Zhang C, Su JY, Hoff CM, Margetts PJ: Prolonged peritoneal gene expression using a helper-dependent adenovirus. Perit Dial Int 2009, 29:508-516.

42. Graham FL, van der Eb AJ: A new technique for the assay of infectivity of human adenovirus 5 DNA. Virology 1973, 52:456-467.

43. Parks RJ, Chen L, Anton M, Sankar U, Rudnicki MA, Graham FL: A helperdependent adenovirus vector system: removal of helper virus by Cremediated excision of the viral packaging signal. Proc Natl Acad Sci USA 1996, 93:13565-13570.

44. Ng P, Parks RJ, Graham FL: Preparation of helper-dependent adenoviral vectors. Methods Mol Med 2002, 69:371-388.

\section{doi:10.1186/1743-422X-10-273}

Cite this article as: Zhou et al:: A novel helper-dependent adenovirusbased cell culture model for Hepatitis $C$ virus replication and production. Virology Journal 2013 10:273. 